Cahiers de recherches médiévales

Journal of medieval studies

L'héritage de Chrétien de Troyes

\title{
Alexandre Micha (1905-2007)
}

Jean Dufournet

\section{OpenEdition}

Journals

Édition électronique

URL : https://journals.openedition.org/crm/2630

DOI : $10.4000 / \mathrm{crm} .2630$

ISSN : 1955-2424

Éditeur

Honoré Champion

Édition imprimée

Date de publication : 15 décembre 2007

Pagination : 1-6

ISSN : 1272-9752

\section{Référence électronique}

Jean Dufournet, «Alexandre Micha (1905-2007) », Cahiers de recherches médiévales [En ligne], 14 |

2007, mis en ligne le 03 mars 2008, consulté le 15 décembre 2022. URL : http://

journals.openedition.org/crm/2630 ; DOI : https://doi.org/10.4000/crm.2630

Ce document a été généré automatiquement le 15 décembre 2022.

Tous droits réservés 


\title{
Alexandre Micha (1905-2007)
}

\author{
Jean Dufournet
}

1 Alexandre Micha allait sur ses cent-deux ans quand il s'est éteint le 31 janvier 2007. L'homme, qui mettait par-dessus tout les qualités de cœur et de courage, s'imposait au respect et à l'amitié par sa modestie et sa rectitude, sa générosité et sa gaieté. Admirateur, lecteur assidu et éditeur de Montaigne, il avait le goût et le sens de la différence à un point tel qu'il aurait pu faire sa devise de ces lignes des Essais : «Pour me sentir engagé à une forme, je n'oblige pas le monde, comme chacun fait, et crois et conçois mille façons de vie ».

2 Sa carrière fut exemplaire à plus d'un titre. D'abord, reçu à l'agrégation en 1929, il commença par faire ses premières armes de professeur dans l'enseignement secondaire pendant vingt ans, à Perpignan, à Bourg-en-Bresse, à Mâcon, à Lyon. Ensuite, il prépara ses thèses de doctorat sur La Tradition manuscrite des romans de Chrétien de Troyes etProlégomènes à une édition de Cligès, soutenues en avril 1939, et ce, tout en enseignant. D'autre part, devenu professeur de l'enseignement supérieur, il connut successivement plusieurs universités : Caen de 1946 à 1948, Strasbourg de 1948 à 1958, puis, de nouveau, Caen et Rouen de 1958 à 1964, Nanterre de 1964 à 1969, enfin la Sorbonne où il termina sa carrière en septembre 1976. Il contribua activement à la création de deux universités nouvelles, celles de Rouen et de Nanterre. Il faut aussi souligner qu'il fut, pour un temps, à la fois médiéviste et seiziémiste, éditeur non seulement des Essais, mais aussi du Second Livre des Amours de Ronsard. Pour finir, notons que sa longue retraite fut très féconde, puisque, de 1978 à 2002, il publia 17 livres, dont la monumentale édition du Lancelot en 9 volumes (1978-1983),et presque autant d'articles.

Il fut un médiéviste de haute volée, un des plus importants, pour ses éditions de Cligès, de Merlin et de Guillaume de Palerne, pour ses traductions (plus de dix) et surtout pour son édition du Lancelot qu'il avait préparée pendant plus de vingt ans, collationnant et confrontant plusde cent manuscrits, éclairant l'œuvre par des articles décisifs (sur la composition, sur la tradition manuscrite, sur l'esprit du Lancelot-Graal, etc.), rassemblés dans l'ouvrage intitulé De la chanson de geste au roman (Genève, Droz, 1976). Ce fut un événement majeur qui tendit à renouveler notre approche et notre connaissance de la littérature médiévale en mettant ànotre disposition un des plus grands textes et, du 
coup, en suscitant de nombreux travaux, thèses et articles, d'autant plus que chaque volume constitue un précieux instrument, de consultation facile, par ses titres courants et ses tables des matières très détaillées, et que le neuvième tome contient un index et un glossaire.

Mais, plutôt que de faire nous-même une présentation détaillée de l'ensemble de l'œuvre ${ }^{1}$, nous avons préféré céder la parole à Alexandre Micha lui-même en reproduisant un entretien que nous avions eu avec lui dans les années $1980^{2}$.

\section{NOTES}

\section{Interview d'Alexandre Micha par Jean Dufournet}

\section{Pourquoi une nouvelle édition du Lancelot?}

Il n'existait du Lancelot jusqu'à ce jour que l'édition d'O. Sommer, aux tomes III-V de sa Vulgate Version of the Arthurian Romances, Washington, 1910-1912. Cette édition a rendu de très grands services. C'est sur elle qu'ont travaillé tous les médiévistes qui, de F. Lot à J. Frappier, se sont intéressés au Lancelot.

Mais ce n'est nullement une édition critique. Sommer a tout simplement recopié, sans même avoir esquissé un classement des manuscrits, le texte d'Additional 10293 $\mathrm{du}$ British Museum. Il n'a d'autre part connu qu'un nombre infime des manuscrits existants, se limitant à ceux qui étaient à sa disposition immédiate, dans le célèbre dépôt anglais, une petite dizaine en tout et pour tout, avec quelques sondages dans le B.N. 339 grâce aux fragments qu'avait publiés Jonckbloet.

En outre, quand son manuscrit le laisse en panne, ce qui arrive fréquemment à partir du deuxième tiers de l'œuvre, il est obligé d'avoir recours à d'autres copies, sous peine d'offrir un texte inintelligible; or tantôt (mais rarement) il signale d'où viennent ses corrections ou ses ajouts, et tous des manuscrits du même British Museum, tantôt il procède à ses émendations sans en indiquer la provenance.

Enfin, par suite des insuffisances de sa documentation - absence d'inventaire des copies, absence de classement - il n'a pas vu que l'on se trouve, à d'importants et très longs passages, en présence de deux versions, une longue et une courte. Quelques notes çà et là, au bas des pages, témoignent bien de ces différences de rédactions, mais de façon exceptionnelle, et plus que fragmentaire.

Ajoutons que l'édition Sommer est depuis longtemps introuvable. Seules les grandes bibliothèques la possèdent, et quelques privilégiés.

Quels principes ont guidé votre édition ? Quelle est votre conception de l'ensemble?

Pour entreprendre une édition critique, il fallait au préalable recenser les manuscrits connus à ce jour. La liste en est longue (et sans doute incomplète dans l'état actuel de 
nos connaissances): complets ou fragmentaires, il n'en existe pas moins d'une centaine.

Le classement a été long à établir. La longueur de l'œuvre nécessitait de nombreux sondages. On distingue non seulement deux versions dont nous venons de parler, une longue, une courte, mais parfois des textes mixtes, ou hybrides, où l'on passe d'une version à l'autre. Un cas frappant est celui de la guerre contre Claudas, un peu avant la fin de l'Agravain, ou encore celui de l'épisode du second voyage en Sorelois et de la fausse Guenièvre où le B.N. 339 commence par donner la version courte, avec le ms. de Rouen, pour passer ensuite sans crier gare à la version vulgate. À l'intérieur des groupes que l'on peut considérer comme relativement stables, la contamination a joué pour brouiller les frontières. Les articles que nous avons publiés dans la Romania en 1964 et 1965 sont le résultat de ces recherches épineuses.

Il fallait de toute évidence, surtout avec une centaine de copies, renoncer à un texte composite, qui eût été un monstre, et un monstre mort-né. Donc choisir un manuscrit de base et le corriger, quand il n'est pas possible de le garder, par les manuscrits apparentés.

Mais le Lancelot est une œuvre très longue, et nous ne connaissons pas de manuscrit qui offre un texte de qualité égale d'un bout à l'autre. Il a fallu, à regret c'est vrai, mais il fallut renoncer par suite à un manuscrit de base unique que l'on aurait suivi du début à la fin : telle copie, excellente dans telle partie, a été ou devient médiocre, voire exécrable, dans ce qui précède ou ce qui suit. Pour la partie qui va du deuxième Voyage en Sorelois jusqu'à l'Agravain, nous avons choisi le ms. de Cambridge, Corpus Christi College 45 ; pour l'Agravain, celui-ci est loin d'être aussi bon : il s'interrompt brusquement au milieu de cette partie et appartient à une famille suspecte: nous avons alors opté pour le Rawlinson 899. C'est un autre manuscrit qui sera la base pour le premier tiers du Lancelot.

Nous avons commencé par la publication du deuxième Voyage en Sorelois, parce qu'à partir de là, la tradition manuscrite est infiniment plus compliquée que dans le premier tiers de l'œuvre. C'est là en effet que se font jour les deux versions : il était par conséquent urgent de débrouiller cet écheveau et de mettre les deux états du texte à la disposition des chercheurs.

L'ensemble de l'édition comprendra neuf volumes. Quatre volumes ont à ce jour paru; le cinquième et le sixième, qui achèveront l'Agravain, sont sous presse; le septième et le huitième reviendront au premier tiers de l'œuvre où la tradition est beaucoup moins embrouillée et où nous n'avons plus qu'une seule version (les mss RN. 110, 111, 112, Bonn 526 abrègent fréquemment, sans constituer pour autant une version différente). Le neuvième volume sera réservé à l'index des noms propres et anonymes et au glossaire.

\section{Que représente ce roman de Lancelot par rapport à Chrétien de Troyes ?}

Pas plus que les autres œuvres médiévales, le Lancelot n'a été créé ex nihilo. Le roman que l'on peut placer vers 1225-1230 reprend une foule de thèmes et de motifs depuis longtemps en circulation, mais il doit beaucoup à Chrétien de Troyes. L'auteur a pris chez le Champenois le thème des amours de Lancelot et de la reine Guenièvre : un long chapitre insère le roman de la Charrette en le dépouillant au reste de la poésie dont avait su l'entourer son devancier. Mais ce qui n'était qu'un épisode, l'enlèvement et la délivrance de Guenièvre par Lancelot chez Chrétien de Troyes, est 
devenu une " estoire » qui nous mène de la naissance de Lancelot et de sa première rencontre avec la reine jusqu'au plein épanouissement sentimental et chevaleresque du héros.

D'autre part, il a combiné la matière du troisième (ou quatrième ?) roman de Chrétien avec celle du Conte $d u$ Graal, ce qui est encore plus vrai du corpus entier (Estoire, Merlin, Lancelot propre,Queste, Mort Artu) que du seul Lancelot propre. De plus en plus, et singulièrement à partir de l'Agravain, le Graal retient l'attention de l'auteur. Gauvain, puis Lancelot, puis Bohort entrent au château de Corbenic où est conservé le SaintVaissel. Chacun d'eux, en présence de la sainte relique, a un comportement propre, de l'indifférence de Gauvain qui n'a d'yeux que pour la belle porteuse, au désir de Bohort de contempler l'insigne merveille. Une discrimination s'établit dès le Lancelot entre les quêteurs : Gauvain est d'ores et déjà disqualifié, alors que Bohort commence à paraître comme ce "saint laborieux » dont parle A. Pauphilet à propos de la Queste. Si Bohort engendre Helain le Blanc dans sa brève rencontre avec la fille du roi Brangoire, Lancelot donne naissance à Galaad grâce à la ruse de Brisane qui le met dans le lit de la fille du roi Pellès, ce qui forme le lien avec la Queste.

C'est dire que, par rapport à Chrétien de Troyes qui s'en tenait, dans la Charrette comme dans le Conte de Graal, à un roman épisodique, le Lancelot appartient à un nouveau type de roman : le roman cyclique. Le procédé de l'entrelacement, amorcé par Chrétien de Troyes, surtout dans son dernier roman, est ici porté à la perfection, et non parfois sans quelque virtuosité gratuite.

Que représente le Lancelot par rapport à la tradition du XIII siècle?

Oui, il faut le replacer dans la tradition du XIII siècle pour en saisir toute l'originalité. D'abord, il inaugure, avec le cycle de Robert de Boron, le roman en prose. Robert l'avait précédé, il est vrai, mais son œuvre originale était en vers avant d'être translatée en prose. C'est à lui en tout cas que l'on devait le nouveau type de roman cyclique, puisqu'il allait des origines du Graal (l'Estoire) à l'annonce du Graal et de la Table Ronde (Merlin) jusqu'à l'accomplissement de la Table Ronde et à la conquête du Graal (Perceval). Cette trilogie (ou tétralogie si l'on se rappelle qu'une brève Mort Artu termine le Perceval de Robert) constituait un devis architectural qu'a amplifié et enrichi l'auteur du Lancelot. L'histoire de l'amour et des exploits progressifs de Lancelot perpétue, mais sur un nouveau plan, le roman d'apprentissage dont il y a des exemples assez nombreux depuis le Conte du Graal. En introduisant l'histoire de Lancelot dans celle du Graal, l'auteur a créé tout un univers où s'affrontent deux idéaux, deux systèmes de valeurs: en face de la spiritualité qu'exaltera la Queste, l'apologie d'un bel amour humain qui ne le cède en rien en grandeur, malgré ses faiblesses, aux élans d'une âme dégagée de toutes attaches charnelles.

Aucune œuvre du XIII ${ }^{e}$ siècle n'a présenté, de surplus, une si grande variété de caractères humains avec les Gauvain, les Claudas, les Hector, les Galaad, les Galehaut, et la dame du Lac, ni provoqué d'aussi longues réflexions sur la grandeur et la servitude de l'amour. Il n'est, pour en sentir la véritable originalité, que de songer aux romans en vers du même temps qui, malgré quelques réussites et mise à part l'œuvre de Jean Renart, rabâchent les merveilles éculées et les quêtes insipides de chevaliers dont les aventures sont sans fin et sans signification. 
Mais cette supériorité du roman en prose ne vient-elle pas, pour une bonne part, de son héros?

Oui, à coup sûr. En effet, les longueurs ne sont pas absentes de ce roman. Quandoque dormitat... On pourra trouver que trop de demoiselles demandent des secours, que trop de coups d'épée, trop de duels au dénouement prévisible occupent trop de pages et que plus d'une fois les aventures se répètent avec quelques variantes qui n'en masquent pas la monotonie. Mais le personnage de Lancelot reste une des grandes créations du roman français. Il s'est imposé tout au long des siècles à l'imagination des générations médiévales, et bien au-delà. Lancelot est devenu le parangon, la "fleur », dit le texte, de toute chevalerie, le modèle du parfait amant. Aucun chevalier ne brille à la cour d'Arthur d'un pareil éclat; nombre d'épisodes n'ont d'autre but que de le montrer, par ses qualités physiques et morales, supérieur aux adversaires de toutes sortes qu'il affronte; il respecte, même au prix d'une certaine cruauté quand il y est obligé, la parole donnée : c'est qu'il a reçu de la bouche de la dame du Lac les commandements du chevalier accompli. Il est aussi le compagnon dévoué de Galehaut, moins absolu toutefois que ce dernier dans les exigences de l'amitié.

Mais il est surtout l'amant de la reine Guenièvre, un amant soumis, corps et âme, aux volontés de sa dame qui est devenue pour lui une sorte de divinité. Ne nous y trompons pas cependant: ce serait une erreur de croire, et bien plus ici encore que pour la Charrette de Chrétien de Troyes, que son personnage n'est qu'une illustration de la fine amor. Certes, Lancelot connaît et observe tous les articles du credo courtois : fidélité, humilité, adoration, dévouement. Mais le personnage est tout autre chose que la mise en œuvre d'une doctrine. Lancelot est un être de chair et de sang. Rien n'est décrit avec plus de finesse que la naissance de son amour, qui va d'un novice à une femme plus âgée, ou que la scène du premier baiser. Lancelot connaît les premiers émois, puis les premières exaltations et bientôt les premières souffrances de l'amour. Le romancier, pour des raisons évidentes, devait le tenir périodiquement éloigné de la cour, dans des prisons ou des errances plus ou moins longues qui devaient d'une part éviter tout scandale et d'autre part lui permettre de prendre conscience de ses véritables sentiments. Cette succession de départs et de retours, d'absences et de désirs comblés constitue le rythme et comme la respiration du roman. Peu à peu, Lancelot découvre qu'il ne restera pas le meilleur chevalier du monde, quels que soient ses exploits, quels que soient ses mérites. Si l'auteur n'est pas porté à l'analyse des sentiments, il a merveilleusement saisi les réactions contradictoires d'un homme que la perfection atteinte ne peut satisfaire pleinement, malgré ses efforts pour s'en persuader. Quelque chose de plus haut demeure pour lui interdit, il est un seuil qu'il ne franchira jamais : ce destin sera réservé à son fils Galaad.

Le Lancelot est l'histoire d'une passion en devenir et non seulement surprise dans un moment de crise : Proust, plutôt que Racine. Le roman ne culmine pas autour d'un ou deux épisodes autour desquels tout s'organise. La durée est sa vraie dimension : la passion s'y développe, s'y approfondit, s'y transforme à travers « frénésies ", doutes, exaltations, dépressions physiques, jalousies, abandons amoureux, et jusqu'au bout cette passion s'entretiendra de son propre feu. Là est la marque qui distingue Lancelot de tous les autres héros de roman.

1. À paraître dans un numéro prochain du Moyen Âge. 
2. Cet interview a déjà paru dans Le Moyen Âge, 87, 1981, p.107-13. 